\title{
On the metric graph model for flows in tubular nanostructures
}

\author{
M. O. Smolkina ${ }^{1}$, I. Y. Popov ${ }^{1}$, I. V. Blinova ${ }^{1}$, E. Milakis $^{2}$ \\ ${ }^{1}$ ITMO University, Kronverkskiy, 49, Saint Petersburg, 197101, Russia \\ ${ }^{2}$ University of Cyprus, P.O.Box 20537, CY-1678 Nicosia, Cyprus \\ vega14@mail.ru, popov1955@gmail.com, irin-a@yandex.ru, emilakis@ucy.ac.cy
}

DOI 10.17586/2220-8054-2019-10-1-6-11

\begin{abstract}
A metric graph model is suggested for the Stokes flow concentrated in the vicinity of a network embedded in $\mathbb{R}^{3}$. As a basic problem, we consider the case corresponding to strong variation of the viscosity and density in a cylinder of small radius. An equation for the main term of the asymptotics is obtained. As for a graph structure, coupling conditions are assumed at the graph vertices.
\end{abstract}

Keywords: stokes flow, metric graph.

Received: 12 November 2018

Revised: 23 January 2019

\section{Introduction}

Many physical systems demonstrate flows concentrated near a network, e.g., flows through nanotubes [1-3] or biophysical flows [4-6]. Mentioned flow concentration near lines or surfaces may be caused by specific variation of viscosity. It is typical, for instance, for geophysical flows [7-10]. Narrowness of the domain means small ratio of width and length. It is worth noting that works concerning to viscous wires with free surface $[11,12]$ are related to glass and fiber technologies. The problems are complicated and it is of great relevance to construct models which allow simplification.

Concentration of a solution near lines or networks appears not only in fluid mechanics but also in other fields, e.g., in quantum theory. There is an effective and simple method for investigating such systems in quantum mechanics - the quantum graph model. This method was developed in the 1980's $([13,14])$ and now is a widely used approach (see, e.g., [15]). The model has some relevant features. On the one hand, it allows one to obtain explicit solutions of the model problem; in addition, it permits good approximation in many particular physical problems (see, e.g., [16-18]). This is one reason for seeking of a new field of application for such an effective instrument. In this paper, we make steps to the development of a model analogs to the quantum graph for fluid mechanics.

There are several ways to consider the hydrodynamic equations on a network (metric graph). One can deal with the 1D Navier-Stokes equation. For example, for compressible fluid of constant viscosity $\widetilde{\eta}$ it takes the form:

$$
\frac{\partial v}{\partial t}+v \frac{\partial v}{\partial x}-\widetilde{\eta} \frac{\partial^{2} v_{x}}{\partial x^{2}}=0
$$

This is a nonlinear equation. To get the corresponding linear one we can linearize it in the vicinity of some solution or simply neglect the nonlinear inertial terms. It is possible to obtain the 1D Navier-Stokes equation by the asymptotic procedure for 3D or 2D Navier-Stokes equations in thin tubes (see, e.g., [19-22]). It is also possible to study linear 2D or 3D Stokes equations in a system of narrow tubes and to consider the limiting procedure for tubes having widths tending to zero. One observes an analogous situation for waveguides and thick quantum graphs (see, e.g., [23-25]). In the most popular model for the Stokes flow in a network one assumes that there is the Poiseuille flow in the tubes [26]). In our case the situation is more complicated (the flow is not localized inside tubes, the viscosity and the density varies essentially), and this model is not appropriate. We use the asymptotic approach. The first step was made in [27], where a metric graph model was constructed for 2D case. As a result, we obtain 1D problem on a metric graph for the main term of the asymptotic expansion. We called it the Stokes graph. In the present paper, we construct the model for 3D case. 


\section{Preliminaries}

3D Stokes and continuity equation for the case of variable viscosity and density in cylindrical coordinates $(r, \varphi, z)$ are as follows:

$$
\begin{gathered}
(\nabla \cdot \sigma)_{r}=-\rho G_{r}, \\
(\nabla \cdot \sigma)_{\phi}=-\rho G_{\phi}, \\
(\nabla \cdot \sigma)_{z}=-\rho G_{z}, \\
\nabla(\rho v)=0 .
\end{gathered}
$$

Here $\sigma$ is the stress tensor, $\rho$ is the density, $G$ is the external force, $v$ is the flow velocity. In cylindrical coordinates the continuity equation (2) takes the form:

$$
\frac{\rho v_{r}}{r}+\frac{\partial\left(\rho v_{r}\right)}{\partial r}+\frac{1}{r} \frac{\partial\left(\rho v_{\phi}\right)}{\partial \phi}+\frac{\partial\left(\rho v_{z}\right)}{\partial z}=0
$$

The divergence of a total stress tensor in cylindrical coordinates have the form:

$$
\begin{gathered}
(\nabla \cdot \sigma)_{r}=\frac{\partial \sigma_{r r}}{\partial r}+\frac{\sigma_{r r}-\sigma_{\phi \phi}}{r}+\frac{1}{r} \frac{\partial \sigma_{\phi r}}{\partial \phi}+\frac{\partial \sigma_{z r}}{\partial z}, \\
(\nabla \cdot \sigma)_{\phi}=\frac{\partial \sigma_{r \phi}}{\partial r}+\frac{2 \sigma_{r \phi}}{r}+\frac{1}{r} \frac{\partial \sigma_{\phi \phi}}{\partial \phi}+\frac{\partial \sigma_{z \phi}}{\partial z} \\
(\nabla \cdot \sigma)_{z}=\frac{\partial \sigma_{r z}}{\partial r}+\frac{\sigma_{r z}}{r}+\frac{1}{r} \frac{\partial \sigma_{\phi z}}{\partial \phi}+\frac{\partial \sigma_{z z}}{\partial z}
\end{gathered}
$$

Then equations (1) transform to the form:

$$
\begin{gathered}
\frac{\partial \tau_{r r}}{\partial r}+\frac{\tau_{r r}-\tau_{\phi \phi}}{r}+\frac{1}{r} \frac{\partial \tau_{r \phi}}{\partial \phi}+\frac{\partial \tau_{r z}}{\partial z}-\frac{\partial P}{\partial r}=-\rho G_{r}, \\
\frac{\partial \tau_{r \phi}}{\partial r}+\frac{2 \tau_{r \phi}}{r}+\frac{1}{r} \frac{\partial \tau_{\phi \phi}}{\partial \phi}+\frac{\partial \tau_{\phi z}}{\partial z}-\frac{1}{r} \frac{\partial P}{\partial \phi}=-\rho G_{\phi}, \\
\frac{\partial \tau_{r z}}{\partial r}+\frac{\tau_{r z}}{r}+\frac{1}{r} \frac{\partial \tau_{\phi z}}{\partial \phi}+\frac{\partial \tau_{z z}}{\partial z}-\frac{\partial P}{\partial z}=-\rho G_{z} .
\end{gathered}
$$

In cylindrical coordinates, the deviatoric stress tensor has the following components:

$$
\begin{gathered}
\tau_{r r}=2 \eta \frac{\partial v_{r}}{\partial r}, \quad \tau_{\phi \phi}=2 \eta\left(\frac{1}{r} \frac{\partial v_{\phi}}{\partial \phi}+\frac{v_{r}}{r}\right), \quad \tau_{z z}=2 \eta \frac{\partial v_{z}}{\partial z} \\
\tau_{r \phi}=\eta\left(\frac{1}{r} \frac{\partial v_{r}}{\partial \phi}+\frac{\partial v_{\phi}}{\partial r}-\frac{v_{\phi}}{r}\right), \quad \tau_{r z}=\eta\left(\frac{\partial v_{z}}{\partial r}+\frac{\partial v_{r}}{\partial z}\right), \\
\tau_{\phi z}=\eta\left(\frac{\partial v_{\phi}}{\partial z}+\frac{1}{r} \frac{\partial v_{z}}{\partial \phi}\right) .
\end{gathered}
$$

In more detail, equations (4), (5), (6) take the form:

$$
\begin{gathered}
2 \frac{\partial \eta}{\partial r} \frac{\partial v_{r}}{\partial r}+2 \eta \frac{\partial^{2} v_{r}}{\partial r^{2}}+2 \eta \frac{1}{r} \frac{\partial v_{r}}{\partial r}-3 \eta \frac{1}{r^{2}} \frac{\partial v_{\phi}}{\partial \phi}-2 \eta \frac{v_{r}}{r^{2}}+\frac{1}{r^{2}} \frac{\partial \eta}{\partial \phi} \frac{\partial v_{r}}{\partial \phi}+ \\
\frac{1}{r} \frac{\partial \eta}{\partial \phi} \frac{\partial v_{\phi}}{\partial r}-\frac{\partial \eta}{\partial \phi} \frac{v_{\phi}}{r^{2}}+\eta \frac{1}{r^{2}} \frac{\partial^{2} v_{r}}{\partial \phi^{2}}+\frac{1}{r} \eta \frac{\partial^{2} v_{\phi}}{\partial \phi \partial r}+ \\
+\frac{\partial \eta}{\partial z} \frac{\partial v_{z}}{\partial r}+\frac{\partial \eta}{\partial z} \frac{\partial v_{r}}{\partial z}+\eta \frac{\partial^{2} v_{z}}{\partial z \partial r}+\eta \frac{\partial^{2} v_{r}}{\partial z^{2}}-\frac{\partial P}{\partial r}=-\rho G_{r} \\
\frac{1}{r} \frac{\partial \eta}{\partial r} \frac{\partial v_{r}}{\partial \phi}+\frac{\partial \eta}{\partial r} \frac{\partial v_{\phi}}{\partial r}-\frac{1}{r} \frac{\partial \eta}{\partial r} v_{\phi}+\eta \frac{1}{r} \frac{\partial^{2} v_{r}}{\partial r \partial \phi}+\eta \frac{\partial^{2} v_{\phi}}{\partial r^{2}}+ \\
3 \eta \frac{1}{r^{2}} \frac{\partial v_{r}}{\partial \phi}+\eta \frac{1}{r} \frac{\partial v_{\phi}}{\partial r}-\eta \frac{v_{\phi}}{r^{2}}+2 \frac{1}{r^{2}} \frac{\partial \eta}{\partial \phi} \frac{\partial v_{\phi}}{\partial \phi}+2 \frac{1}{r^{2}} \frac{\partial \eta}{\partial \phi} v_{r}+ \\
2 \eta \frac{1}{r^{2}} \frac{\partial^{2} v_{\phi}}{\partial \phi^{2}}+\frac{\partial \eta}{\partial z} \frac{\partial v_{\phi}}{\partial z}+\frac{1}{r} \frac{\partial \eta}{\partial z} \frac{\partial v_{z}}{\partial \phi}+\eta \frac{\partial^{2} v_{\phi}}{\partial z^{2}}+\eta \frac{1}{r} \frac{\partial^{2} v_{z}}{\partial z \partial \phi}-\frac{1}{r} \frac{\partial P}{\partial \phi}=-\rho G_{\phi}, \\
\frac{\partial \eta}{\partial r} \frac{\partial v_{z}}{\partial r}+\frac{\partial \eta}{\partial r} \frac{\partial v_{r}}{\partial z}+\eta \frac{\partial^{2} v_{z}}{\partial r^{2}}+\eta \frac{\partial^{2} v_{r}}{\partial r \partial z}+\eta \frac{1}{r} \frac{\partial v_{z}}{\partial r}+\eta \frac{1}{r} \frac{\partial v_{r}}{\partial z}+\frac{1}{r} \frac{\partial \eta}{\partial \phi} \frac{\partial v_{\phi}}{\partial z} \\
+\frac{1}{r^{2}} \frac{\partial \eta}{\partial \phi} \frac{\partial v_{z}}{\partial \phi}+\eta \frac{1}{r} \frac{\partial^{2} v_{\phi}}{\partial \phi \partial z}+\eta \frac{1}{r^{2}} \frac{\partial^{2} v_{z}}{\partial \phi^{2}}+2 \eta \frac{\partial^{2} v_{z}}{\partial z^{2}}+2 \frac{\partial \eta}{\partial z} \frac{\partial v_{z}}{\partial z}-\frac{\partial P}{\partial z}=-\rho G_{z} .
\end{gathered}
$$




\section{Stokes graph model}

We will consider the flow under some axisymmetric conditions. Keeping in mind future replacement of variables, we change notations. Namely, we equip all variables and parameters in equations (3), (7), (8), (9) by $\widetilde{r}, \widetilde{\varphi}, \widetilde{z}, \widetilde{\eta}, \widetilde{\rho}$. We will deal with the case when the values of the viscosity $\widetilde{\eta}$ and the density $\widetilde{\rho}$ are smooth and outside the cylinder $\{(\widetilde{r}, \widetilde{\varphi}, \widetilde{z}): \widetilde{z} \in(-\infty, \infty), \widetilde{r} \in(0, \varepsilon)\}$ are essentially greater than inside it $((\widetilde{r}, \widetilde{\varphi}, \widetilde{z})$ are the cylindrical coordinates):

$$
\begin{aligned}
\widetilde{\eta}(\widetilde{r}, \widetilde{\varphi}, \widetilde{z}) & =\eta(\widetilde{z}) \eta_{2}(\widetilde{r}), \quad \widetilde{\rho}(\widetilde{r}, \widetilde{\varphi}, \widetilde{z})=\rho(\widetilde{z}) \rho_{2}(\widetilde{r}), \\
\eta_{2}(\widetilde{r}) & =\left\{\begin{array}{cc}
1, & 0 \leq \widetilde{r} \leq \varepsilon, \\
\eta_{w}, & \widetilde{r}>2 \varepsilon,
\end{array} \eta_{w} \gg 1,\right. \\
\rho_{2}(\widetilde{r}) & =\left\{\begin{array}{cc}
1, & 0 \leq \widetilde{r} \leq \varepsilon, \\
\rho_{w}, & \widetilde{r}>2 \varepsilon,
\end{array} \quad \rho_{w} \gg 1 .\right.
\end{aligned}
$$

Here, $\varepsilon$ is a small parameter. It means that the flow is essentially inside the cylinder. We will consider the flow inside the cylinder only. Let us introduce new coordinates: $z=\widetilde{z}, r=\widetilde{r} / \varepsilon, \varphi=\widetilde{\varphi}$. We will consider an axisymmetric solution. Correspondingly, the flow velocity and the pressure do not depend on $\varphi$. Correspondingly, equation (8) becomes the identity. Equations (7), (9), (3) take the following form after the coordinate replacement:

$$
\begin{gathered}
2 \varepsilon^{-2} \frac{\partial \eta}{\partial r} \frac{\partial v_{r}}{\partial r}+2 \varepsilon^{-2} \eta \frac{\partial^{2} v_{r}}{\partial r^{2}}+2 \varepsilon^{-2} \eta \frac{1}{r} \frac{\partial v_{r}}{\partial r}-2 \varepsilon^{-2} \eta \frac{v_{r}}{r^{2}}+\varepsilon^{-1} \frac{\partial \eta}{\partial z} \frac{\partial v_{z}}{\partial r}+ \\
\varepsilon^{-1} \frac{\partial \eta}{\partial z} \frac{\partial v_{r}}{\partial z}+\varepsilon^{-1} \eta \frac{\partial^{2} v_{z}}{\partial r \partial z}+\eta \frac{\partial^{2} v_{r}}{\partial z^{2}}-\varepsilon^{-1} \frac{\partial P}{\partial r}=-\rho G_{r} \\
\varepsilon^{-2} \frac{\partial \eta}{\partial r} \frac{\partial v_{z}}{\partial r}+\varepsilon^{-1} \frac{\partial \eta}{\partial r} \frac{\partial v_{r}}{\partial z}+\varepsilon^{-2} \eta \frac{\partial^{2} v_{z}}{\partial r^{2}}+\varepsilon^{-1} \eta \frac{\partial^{2} v_{r}}{\partial z \partial r}+\varepsilon^{-2} \eta \frac{1}{r} \frac{\partial v_{z}}{\partial r}+ \\
\varepsilon^{-1} \eta \frac{1}{r} \frac{\partial v_{r}}{\partial z}+2 \eta \frac{\partial^{2} v_{z}}{\partial^{2} z}+2 \frac{\partial \eta}{\partial z} \frac{\partial v_{z}}{\partial z}-\frac{\partial P}{\partial z}=-\rho G_{z} \\
\varepsilon^{-1} \frac{\rho}{r} v_{r}+\varepsilon^{-1} \frac{\partial \rho}{\partial r} v_{r}+\varepsilon^{-1} \rho \frac{\partial v_{r}}{\partial r}+\frac{\partial\left(\rho v_{z}\right)}{\partial z}=0 .
\end{gathered}
$$

We will seek the solutions in the form of series in $\varepsilon$ :

$$
v_{r}=v_{r}^{0}+v_{r}^{1} \varepsilon+\ldots, v_{z}=v_{z}^{0}+v_{z}^{1} \varepsilon+\ldots, P=P^{0}+P^{1} \varepsilon+\ldots
$$

Let us insert the series (3) into equations (10), (11), (12) and collect terms of the same powers of $\varepsilon$. We obtain the chain of equations for the series coefficients. Terms of order $\varepsilon^{-2}$ are in the Stokes equations (in new variables) only:

Equation (14) gives us:

$$
\begin{gathered}
\eta \frac{\partial^{2} v_{r}^{0}}{\partial r^{2}}+\frac{1}{r} \frac{\partial(r \eta)}{\partial r} \frac{\partial v_{r}^{0}}{\partial r}-\frac{\eta}{r^{2}} v_{r}^{0}=0 \\
\eta \frac{\partial^{2} v_{z}^{0}}{\partial r^{2}}+\frac{1}{r} \frac{\partial(r \eta)}{\partial r} \frac{\partial v_{z}^{0}}{\partial r}=0 .
\end{gathered}
$$

$$
v_{z}^{0}(r, z)=\frac{f(z)}{\eta(z)} \int \frac{d r}{r \eta_{2}(r)}=u(z) \int \frac{d r}{r \eta_{2}(r)} .
$$

Here $u(z), f(z)$ are some functions of one variable. They should be determined later. Terms of order $\varepsilon^{-1}$ are in three equations. The continuity equation gives us: $v_{r}^{0}=g(z) /(r \rho)$. To avoid contradiction with (13), we conclude that $v_{r}^{0}=0$, i.e. the series for $v_{r}$ starts from another power than for $v_{z}$. It is a conventional situation when one has different scales for different directions (see, e.g., [31]). The Stokes equations (10), (11) give us the following relations for the terms of this order:

$$
\begin{gathered}
\eta \frac{\partial^{2} v_{r}^{1}}{\partial r^{2}}+\frac{1}{r} \frac{\partial}{\partial r}(r \eta) \frac{\partial v_{r}^{1}}{\partial r}-\frac{\eta}{r^{2}} v_{r}^{1}=-\frac{\partial \eta}{\partial z} \frac{\partial v_{z}^{0}}{\partial r}-\eta \frac{\partial^{2} v_{z}^{0}}{\partial r \partial z}+\frac{\partial P^{0}}{\partial r} \\
\frac{\eta}{\partial r} \frac{\partial v_{z}^{1}}{\partial r}+\eta \frac{\partial^{2} v_{z}^{1}}{\partial^{2} r}+\frac{1}{r} \frac{\partial v_{z}^{1}}{\partial r}=0
\end{gathered}
$$

These equations are solvable with respect to $v_{r}^{1}, v_{y z}^{1}$. The pressure term $P^{0}$ should be determined later.

Consider the terms of order $\varepsilon^{0}$. The continuity equation (12) leads to the following equation for $v_{r}^{1}$ :

$$
\frac{\partial \rho}{\partial r} v_{r}^{1}+\frac{\rho}{r} v_{r}^{1}+\rho \frac{\partial v_{r}^{1}}{\partial r}=-\frac{\partial \rho}{\partial z} v_{z}^{0}-\rho \frac{\partial v_{z}^{0}}{\partial z}
$$


Equation (10) gives the relation:

Equation (11) produces the relation:

$$
\begin{gathered}
2 \eta \frac{\partial^{2} v_{r}^{2}}{\partial r^{2}}+2 \frac{\partial \eta}{\partial r} \frac{\partial v_{r}^{2}}{\partial r}+2 \frac{\eta}{r} \frac{\partial v_{r}^{2}}{\partial r}-2 \eta \frac{v_{r}^{2}}{r^{2}}= \\
-\frac{\partial \eta}{\partial z} \frac{\partial v_{z}^{1}}{\partial r}-\frac{\partial \eta}{\partial z} \frac{\partial v_{r}^{1}}{\partial z}-\eta \frac{\partial^{2} v_{z}^{1}}{\partial z \partial r}+\frac{\partial P^{1}}{\partial r}-\rho G_{r}
\end{gathered}
$$

$$
\begin{gathered}
\eta \frac{\partial^{2} v_{z}^{2}}{\partial r^{2}}+\frac{\partial \eta}{\partial r} \frac{\partial v_{z}^{2}}{\partial r}+\frac{\eta}{r} \frac{\partial v_{z}^{2}}{\partial r}= \\
-\frac{\partial \eta}{\partial r} \frac{\partial v_{r}^{1}}{\partial z}-\frac{\partial \eta}{\partial z} \frac{\partial v_{z}^{0}}{\partial z}-\eta \frac{\partial^{2} v_{r}^{1}}{\partial z \partial r}-\frac{\eta}{r} \frac{\partial v_{z}^{1}}{\partial z}-2 \eta \frac{\partial^{2} v_{z}^{0}}{\partial z^{2}}+\frac{\partial P^{0}}{\partial r z}-\rho G_{z} .
\end{gathered}
$$

The pressure is determined at the next stage (by taking into account the solvability condition $\left(\frac{\partial^{2} P^{0}}{\partial r \partial z}=\frac{\partial P^{0}}{\partial z \partial r}\right)$ where the right hand side is obtained as a derivative with respect to $r$ of the expression obtained from (18) and the left hand side - as a derivative in respect to $z$ of the expression obtained from (17)). If we consider the first term of the asymptotic series for the velocity only, we obtain an interesting equation for $v_{z}^{0}$ from (18) and (16):

$$
\frac{\partial^{2} v_{z}^{0}}{\partial z^{2}}-\frac{\partial \eta}{\partial z} \frac{1}{\eta} \frac{\partial \rho}{\partial z} \frac{1}{\rho} v_{z}^{0}=-\frac{\rho}{\eta} G_{z}+\frac{1}{\eta} \frac{\partial P^{0}}{\partial z} .
$$

Taking into account the expressions for $\eta, \rho, v_{z}^{0}$, one can see that it leads to the following equation for the function $u(z)$ in the homogeneous case $\left(G_{x}=0, \quad \frac{\partial P^{0}}{\partial z}=0\right)$ :

$$
u^{\prime \prime}-\frac{\eta^{\prime}}{\eta} \frac{\rho^{\prime}}{\rho} u=0
$$

One can see that we have the 1D Schrödinger equation with a specific potential for the function $u(x)$ corresponding to zero energy (the similar problem for the quantum graph is known as the threshold resonance, see, e.g., [28]).

Thus, for the flow concentrated near a line (a thin cylinder) we have one-dimensional model. Correspondingly, more complicated case of flow concentrated near a network (system of coupled segments) can be reduced to the corresponding metric graph with the Schrödinger operator:

$$
H=-\frac{d^{2}}{d x^{2}}+\frac{\eta^{\prime}}{\eta} \frac{\rho^{\prime}}{\rho}
$$

on the edges as the first approximation for the description of the flow. We call it the Stokes graph $(\Gamma)$. It is necessary to determine boundary conditions at the graph vertices. Consider a vertex (let it be zero point) with $n$ output edges. From physical conditions, one has:

and

$$
\rho_{1}(0)=\rho_{2}(0)=\cdots \rho_{n}(0)=\rho(0)
$$

$$
u_{1}^{\prime}(+0)=u_{2}^{\prime}(+0)=\cdots u_{n}^{\prime}(+0)=u^{\prime}(0)
$$

Here indices mark the edges $\left(u_{j}\right.$ is the value of $u$ at $j$-th edge). The last condition is related to the pressure continuity (see, e.g., $[26,29,30]$ ). Here, $u_{j}{ }^{\prime}(+0)$ is the derivative in the outgoing direction at the vertex 0 . The continuity equation gives us for this vertex:

$$
\sum_{j=1}^{n} u_{j}=-\left(\frac{\rho(0)}{\sum_{j=1}^{n} \rho_{j}^{\prime}(+0)}\right) u^{\prime}(0) .
$$

It is similar to well-known $\delta^{\prime}$-coupling condition for the quantum graph [15]. The coupling constant is related to the density derivative.

We will consider the following case: $q=\frac{\eta^{\prime}}{\eta} \frac{\rho^{\prime}}{\rho}$ is uniformly continuous on every edge of the graph $\Gamma$ (we mark the set of its edges as $E$ and the set of its vertices as $V$ ),

$$
\theta=\frac{\rho(0)}{\sum_{j=1}^{n} \rho_{j}^{\prime}(+0)}
$$

is positive. We will deal with the following homogeneous equation:

$$
(H u)(x)=0 .
$$


Here, operator $H$ is defined as follows. At each edge it acts as:

$$
(H u)(x)=-\frac{d^{2}}{d x^{2}} u(x)+q(x) u(x), x \in E(\Gamma) .
$$

Elements from its domain belong to the Sobolev space $H^{2}$ at each edge. As for vertices, the following condition takes place (for a vertex $x$ having $n$ output edges):

$$
\begin{gathered}
u_{1}{ }^{\prime}(+x)=u_{2}{ }^{\prime}(+x)=\cdots u_{n}{ }^{\prime}(+x)=u^{\prime}(x), x \in V(\Gamma), \\
\sum_{j=1}^{n} u_{j}(x)+\theta u^{\prime}(x)=0, x \in V(\Gamma), \theta>0 . \\
u(a)=0, a \in \partial \Gamma .
\end{gathered}
$$

One can see that we have constructed a metric graph model which coincides with that of the two-dimensional case [27]. Of course, the definition of the function $u$ differs from that of the $2 \mathrm{D}$ case but the model operator is the same. Correspondingly, one can obtain the same result for "cylindrical" case. Namely, we obtain the following statement.

Definition. Operator $H$ is denoted as sign preserving on the graph $\Gamma$ if the inequality $u(x) u\left(x^{\prime}\right)>0$ takes place for any nontrivial solution of equation (22). Here $x, x^{\prime}$ are arbitrary points of $\Gamma \backslash \partial \Gamma$.

The main result of the paper is the following inequality (27) that is analogous to the Harnack's inequality for an elliptic operator on a manifold.

Main Theorem. Let $H$ be sign preserving on graph $\Gamma$. Then there exists a constant $\gamma$, determined by the operator $H$ and the structure of the graph $\Gamma$ only, such that each non-negative on $\Gamma$ solution $u(x)$ of inequality $H u \geq 0$ satisfies the following inequality:

$$
\max _{x \in \Gamma_{0}} u(x) \leq \gamma \min _{x \in \Gamma_{0}} u(x)
$$

on any locally compact (in respect to $\Gamma$ ) subgraph $\Gamma_{0}, \Gamma_{0} \subset \Gamma$.

\section{Acknowledgements}

This work was partially financially supported by the Government of the Russian Federation (grant 08-08), by grant 16-11-10330 of Russian Science Foundation. E. Milakis was partially supported by Research Promotion Foundation (Cyprus) grant no Excellence/1216/0025.

\section{References}

[1] Chivilikhin S.A., Gusarov V.V., Popov I.Y. Charge pumping in nanotube filled with electrolyte. Chinese Journal of Physics, 2018, 56(5), P. 2531-2537.

[2] Popov I.Yu., Kyzyurova K.N. and Blinova I.V., Stokes flow driven by a Stokeslet in a cone. Acta Mechanica, 2014,225 , P. $3115-3121$.

[3] Belonenko M.B., Chivilikhin S.A., Gusarov V.V., Popov I.Yu. and Rodygina O.A. Soliton-induced flow in carbon nanotube. Europhys. Lett., 2013, 101(6), P. 66001.

[4] Sharan M. and Popel A.S. A two-phase model for flow of blood in narrow tubes with increased effective viscosity near the wall. Biorheology, 2001, 38, P. 415-428.

[5] Secomb T.W. and El-Kareh A.V. Models for slow blood flow in narrow tubes: effect of aggregation and sedimentation on flow resistance. Biorheology, 1995, 32, P. 169-169.

[6] Srivastava V.P. A theoretical model for blood flow in small vessels. Int. J. Applications and Appl. Math., 2007, 2(1), P. 51-65.

[7] Gerya T. Introduction to Numerical Geodynamic Modelling. Cambridge, Cambridge University Press, 2010.

[8] Ismail-Zadeh A., Tackley P. Computational methods in Geodynamics. Cambridge, Cambridge University Press, 2010.

[9] Ribe N.M., et al. Buckling instabilities of subducted lithosphere beneath the transition zone. Earth and Planetary Science Letters, 2007, 254, P. 173-179.

[10] Griffiths R.W. The Dynamics of Lava Flows. Annual Review of Fluid Mechanics, 2000, 32(1), P. $477-518$.

[11] Griffiths I.M., Howell P.D. The surfacetension-driven evolution of a two-dimensional annular viscous tube. Journal of Fluid Mechanics, 2007, 593, P. 181-208.

[12] Griffiths I.M., Howell P.D. Mathematical modelling of non-axisymmetric capillary tube drawing. Journal of Fluid Mechanics, 2008, 605, P. 181-206.

[13] Gerasimenko N., Pavlov B. Scattering problems on non-compact graph. Theor. Math. Phys., 1988, 74, P. $230-240$.

[14] Exner P. and Seba P. Free quantum motion on a branching graph. Rep. Math. Phys., 1989, 28, P. 7-26.

[15] Berkolaiko G., Kuchment P., Introduction to Quantum Graphs. AMS, Providence, 2012.

[16] Kostrykin V., Schrader R. Laplacians on metric graphs: eigenvalues, resolvents and semigroups. Quantum graphs and their applications, 201-225, Contemp. Math., 415, Amer. Math. Soc., Providence, RI, 2006.

[17] Popov I.Yu., Skorynina A.N., Blinova I.V. On the existence of point spectrum for branching strips quantum graph. J. Math. Phys., 2014, 55, P. 033504/1-20.

[18] Eremin D.A., Grishanov E.N., Nikiforov D.S., Popov I.Y. Wave dynamics on time-depending graph with Aharonov-Bohm ring. Nanosystems: Physics, Chemistry, Mathematics, 2018, 9(4), P. 457-463. 
[19] Panasenko G.P. Asymptotic expansion of the solution of Navier-Stokes equation in tube structure and partial asymptotic decomposition of the domain. Applicable Analysis, 2000, 76(3), P. 363-381.

[20] Panasenko G.P., Stavre R. Asymptotic analysis of a periodic flow in a thin channel with visco-elastic wall. J. Math. Pures Appl., 2006, 85, P. 558-579.

[21] Panasenko G.P., Stavre R. Asymptotic analysis of the Stokes flow in a thin cylindrical elastic tube. Applicable Analysis, 2012, 91(11), P. 1999-2027.

[22] Melikhov I.F., Popov I.Y. Asymptotic analysis of thin viscous plate model. Nanosystems: Physics, Chemistry, Mathematics, 2018, 9(4), P. 447-456.

[23] Post O. Branching quantum waveguides with Dirichlet boundary conditions: the decoupling case. J. Phys. A: Math. Gen., 2005, 38, P. 4917-4931.

[24] Molchanov S., Vainberg B. Scattering solutions in networks of thin fibers: small diameter asymptotics. Comm. Math.Phys., 2007, 273, P. 533-559.

[25] Popov I.Yu., Eremin D.A., Ivanov D.A. Regular potential approximation for delta-perturbation supported by curve of the Laplace-Beltrami operator on the sphere. Z. Anal. Anwen., 2012, 31(2), P. 125-137.

[26] Jivkov A.P., Hollis C., Etiese F., McDonald S.A., Withers P.J. A novel architecture for pore network modelling with applications to permeability of porous media. J. Hydrology, 2013, 486, P. 246-258.

[27] Kovaleva M.O., Popov I.Y. Harnack's Inequality for Stokes Graph. Zeitschrift fur Analysis und ihre Anwendungen, 2016, 35(4), P. 383-396.

[28] Cacciapuoti C. and Exner P. Nontrivial edge coupling from a Dirichlet network squeezing: the case of a bent waveguide. J. Phys. A., 2007, 40, P. F511-F523.

[29] Gudzovski A.V. On the computation for hydraulic networks. Doklady Akad. Nauk, 1998, 358, P. $765-767$.

[30] Pokornyi Yu.V., Penkin O.M., Pryadev V.L., Borovskikh A.V., Lazarev K.P. and Shabrov S.A. Differential Equations on Geometrical Graphs. Moscow, Fizmatlit, 2004.

[31] Van Dyke M. Perturbation Methods in Fluid Mechanics. Stanford, Parabolic Press, 1975. 